\title{
Effet du poids de l'œuf de pintade (Numida meleagris) sur les paramètres de reproduction et de croissance des pintadeaux au Burkina Faso
}

\author{
Rahamane Sanfo ${ }^{1 *}$ Fabiola Traoré ${ }^{1}$ Bernadette Yougbare ${ }^{1}$ \\ Weremé Ouali ${ }^{1}$
}

Mots-clés

Numida meleagris, pintade, caractéristique de l'œuf, éclosabilité, fertilité, croissance, Burkina Faso

\footnotetext{
Submitted: 24 April 2015

Accepted: 5 April 2018

Published: 23 April 2018

DOI : $10.19182 /$ remvt.31527
}

\begin{abstract}
Résumé
Deux mille trois cents œufs de pintades collectés à la station de Gampéla au Burkina Faso ont été pesés puis répartis en six catégories allant de 20-25 g à 45-50 g. Les œufs ont été incubés et les paramètres suivants ont été enregistrés : les taux de fertilité et d'éclosion, le poids des pintadeaux, les mortalités, et la croissance pondérale jusqu'à 56 jours d'âge. Le poids moyen des œufs a été de $35,2 \pm 1,3 \mathrm{~g}$ avec une répartition inégale dans les six catégories; le plus grand nombre d'œufs était dans la catégorie des 35-40 g. Le taux de fertilité moyen des œufs a été de $84,4 \%$; il était corrélé $(r=0,81 ; p<0,05)$ au poids de l'œuf. Le taux d'éclosion apparent a été de $65,7 \%$ et le taux réel de $82,7 \%$; ce dernier était corrélé $(r=0,85 ; p<0,05)$ au poids de l'œuf. Le poids vif moyen du pintadeau à l'éclosion a été de $25,3 \pm 4,3 \mathrm{~g}$; il était également significativement lié au poids de l'œuf $(r=0,96 ; p<0,05)$. Le taux de mortalité des pintadeaux a été en moyenne de $16,5 \%$; il était lié au poids de l'œuf et donc du pintadeau. Le poids moyen des pintadeaux à 56 jours (PDS56) a été de 152,5 $\pm 20,3 \mathrm{~g}$; il était lié au poids de l'œuf (PO) selon la régression : PDS56 $=-279,1+11,8$ PO avec $R^{2}=0,87$. Cette étude montre que dans la perspective de production de pintadeaux performants il importe de rejeter les œufs de calibre faible $(<35 \mathrm{~g})$.

- Comment citer cet article : Sanfo R., Traoré F., Yougbare B., Ouali W., 2017. Effect of the egg weight of guinea fowl (Numida meleagris) on growth and reproduction parameters of chicks in Burkina Faso. Rev. Elev. Med. Vet. Pays Trop., 70 (4): 121-125, doi: 10.19182/remvt.31527
\end{abstract}

\section{INTRODUCTION}

Au Burkina Faso, l'élevage de la pintade locale a une grande importance et de grandes opportunités de développement. En revanche, cette volaille n'a pas encore fait l'objet d'études approfondies tant de la part des techniciens de développement rural que de celle de

1. Institut de l'environnement et de recherches agricoles (INERA), Direction régionale de l'environnement agricole et de formation de Kamboinsé, 04 BP 8645, Ouagadougou 04, Burkina Faso.

* Auteur pour la correspondance Email :r_sanfo@yahoo.fr la recherche dans ce pays. L'absence de recommandations techniques adaptées restreint la valorisation de cette production et de nombreuses contraintes limitent les producteurs dans leurs activités.

L'un des problèmes importants de l'élevage de pintades au Burkina Faso est le taux de mortalité très élevé des pintadeaux, de $73 \%$ à $100 \%$ (Bessin et al., 1998 ; Hien et al., 2002). Les performances de reproduction (fertilité, fécondité) sont également souvent déficientes (Alkan et al., 2008 ; Ayorinde et al., 1988). En outre l'hétérogénéité des performances est également préjudiciable à l'élevage (Ayorinde et al., 1986). Ces facteurs peuvent être liés aux caractéristiques des œufs. Chez la poule, Ramaphala et Mbajiorgu (2013) rapportent une corrélation négative entre la fertilité des œufs et leurs dimensions. Ces résultats sont confirmés par Sahin et al. (2009), contrairement aux 
résultats de Caglayan et al. (2009). Senapati et al. (1996) notent dans leur étude sur les poules une corrélation positive entre les dimensions des œufs et l'éclosabilité. Quant à Wilson (1991), tout en soulignant l'importance économique du poids de l'œuf, il établit une corrélation positive entre celui-ci et le poids des poussins à l'éclosion.

La relation entre le poids de l'œuf et les paramètres de reproduction chez la pintade a été étudiée au Botswana par Moreki et Mothei (2013) mais elle doit être précisée dans d'autres conditions comme celles du Burkina Faso. Notre étude a ainsi eu pour objectif de déterminer l'effet du poids de l'œuf de la pintade locale sur les paramètres de reproduction et de croissance ultérieure des pintadeaux, au centre du Burkina Faso.

\section{MATERIEL ET METHODES}

L'essai a été réalisé sur 2300 œufs de pintades de souche locale, élevées à la station de Gampéla de l'Institut de développement rural près de Ouagadougou au Burkina Faso. Les œufs ont été conservés au maximum pendant une semaine à une température de $15^{\circ} \mathrm{C}$, pesés (balance de portée $1 \mathrm{~kg}$ et de sensibilité $\pm 0,001 \mathrm{~g}$ ) et répartis en fonction de leur poids en six catégories : de $20 \mathrm{à} 50 \mathrm{~g}$ par pas de $5 \mathrm{~g}$.

Les œufs ont été placés dans un incubateur (type 2-500 FH, Maino Enrico, Oltrona, Italie) d'une capacité de 2500 œufs. La température et l'hygrométrie relative de l'incubateur étaient respectivement de l'ordre de $38,2^{\circ} \mathrm{C}$ et de $60 \%$ (Ayorinde et al., 1988), et l'angle de retournement automatique des œufs a été fixé à $45^{\circ}$. Après 23 jours d'incubation, les œufs ont été transférés dans un éclosoir où la température était de $37^{\circ} \mathrm{C}$ et l'humidité relative de $90 \%$.

Le taux de fertilité a été déterminé par mirage au neuvième jour après incubation. Le taux moyen apparent d'éclosion se calcule par: nombre total d'œufs éclos / nombre total d'œufs incubés x 100. Et le taux moyen réel d'éclosion s'obtient ainsi : nombre d'œufs éclos / nombre d'œufs fertiles x 100.

A deux jours d'âge, les pintadeaux ont été identifiés par des bagues alaires, gardés dans des éleveuses munies d'une lampe tempête à une température avoisinant $38^{\circ} \mathrm{C}$ jusqu'à huit semaines d'âge. A partir de la neuvième semaine, ils ont été élevés au sol avec une densité environ d'un individu par mètre carré. La prophylaxie médicale a été assurée à la troisième semaine par un déparasitage puis par une vaccination contre la maladie de Newcastle. Les pintadeaux ont été nourris avec des aliments industriels standard, abreuvés puis gardés selon la catégorie du poids de l'œuf.

Les animaux ont été pesés individuellement tous les 15 jours (balance EKS de portée $5 \mathrm{~kg}$ et de sensibilité $5 \mathrm{~g}$ ). Les mortalités des pintadeaux ont été enregistrées tous les jours, de l'éclosion à huit mois d'âge, selon la catégorie de poids des œufs.
Les saisies des données ont été effectuées à l'aide du logiciel Excel (2003). Les analyses de variance et la comparaison des moyennes (présentées sous forme de moyenne \pm écart-type) ont été faites à l'aide du logiciel Stat View (version 4.5, SAS Institute, Cary, NC, USA). La comparaison des moyennes (au seuil de $5 \%$ ) a été faite par le test t de Student. Les corrélations entre phénomènes ont été analysées au niveau de signification de $5 \%$ par le test $\mathrm{Z}$.

\section{RESULTATS ET DISCUSSION}

\section{Poids de l'œuf}

Le poids moyen des œufs a été de 35,2 $\pm 1,3 \mathrm{~g}$. La répartition par catégorie de poids est indiquée dans le tableau I. La catégorie la plus représentée était celle des 35-40 g (49,0\% des œufs), et secondairement des 40-45 g (24,4\%) et 30-35 g (19,2\%). Ces trois catégories représentaient au total $92,60 \%$ des œufs. Il y a eu seulement 18 œufs de moins de $20 \mathrm{~g}(0,8 \%)$. Le poids moyen de l'œuf $(37,3 \pm 4,1 \mathrm{~g})$ a été comparable à celui de 35,7 $\pm 2,8 \mathrm{~g}$ rapporté par Hien (2002) à l'ouest du Burkina Faso, et à celui de 37,3 g noté par Kuit al. (1986) au Mali central. Il a été légèrement inférieur à l'intervalle de 35,8-44,6 g noté par Ayorinde et al. (1988) au Nigeria.

\section{Taux de fertilité et d'éclosion}

Le taux moyen de fertilité des œufs a été de 70,3\% pour l'ensemble des six catégories. Ce taux a atteint $84,4 \%$ sans la catégorie des œufs les plus légers (20-25 g) qui ont enregistré un taux de fertilité nul (tableau II). Le taux de fertilité a augmenté avec la catégorie du poids de l'œuf (de $0,0 \%$ à 92,5\%). Il était significativement ( $\mathrm{r}=0,81$, $\mathrm{p}<0,05)$ corrélé au poids de l'œuf.

Les éclosions se sont étalées pendant trois jours du $26^{\mathrm{e}}$ au $28^{\mathrm{e}}$ jour de la mise en incubation des œufs. Le maximum d'éclosions (80,7\%) a été relevé le 27 jour. Le taux réel d'éclosion a été significativement corrélé $(r=0,85, \mathrm{p}<0.05)$ avec le poids de l'œuf.

Les œufs de la catégorie des 20-25 g ont été soustraits de l'incubation par suite de l'absence d'œufs fertiles. Le taux moyen apparent d'éclosion a été de $65,7 \%$ pour l'ensemble des œufs. Le taux moyen réel d'éclosion a été de $82,7 \%$ (tableau II).

Un faible taux d'éclosion n'a pas été observé pour les œufs de poids moyen de 45-50 g, comme rapporté par Hien et al. (2000). Les résultats relatifs au taux d'éclosion de cette catégorie d'œufs sont en accord avec ceux de ministère de l'Economie et des Finances (MEF, 2011). Selon celui-ci, le taux d'éclosion élevé des œufs lourds s'explique par la plus grande épaisseur de leur coquille et l'importance des réserves vitellines. Les œufs lourds de la population de pintades étudiées présentaient vraisemblablement ces caractéristiques.

\section{Tableau I}

Poids moyen de l'œuf de pintade (Numida meleagris) selon la catégorie

\begin{tabular}{lccccccc} 
Catégorie & $\mathbf{2 0 - 2 5}$ & $\mathbf{2 5 - 3 0}$ & $\mathbf{3 0 - 3 5}$ & $\mathbf{3 5 - 4 0}$ & $\mathbf{4 0 - 4 5}$ & $\mathbf{4 5 - 5 0}$ & Ensemble \\
\hline $\begin{array}{l}\text { Nb. d'œufs } \\
\text { \% d'œufs }\end{array}$ & 18 & 108 & 458 & 1127 & 561 & 28 & 2300 \\
& 0,8 & 4,7 & 19,2 & 49,0 & 24,4 & 1,2 & 100 \\
Poids (g) & $22,5^{\mathrm{a}}$ & $28,1^{\mathrm{b}}$ & $33,2^{\mathrm{c}}$ & $37,7^{\mathrm{d}}$ & 41,7 e & $46,3^{\mathrm{f}}$ & 37,3 \\
\pm écart-type & $\pm 1,2$ & $\pm 1,3$ & $\pm 1,3$ & $\pm 1,4$ & $\pm 1,4$ & $\pm 0,9$ & $\pm 4,1$
\end{tabular}

Les différences entre les valeurs suivies de la même lettre en exposant sur la même ligne ne sont pas significatives au seuil de $5 \%$. 
Tableau II

Taux d'œufs clairs et d'œufs fertiles selon la catégorie de poids de l'œuf de pintade (Numida meleagris)

\begin{tabular}{|c|c|c|c|c|c|c|c|c|}
\hline \multicolumn{2}{|l|}{ Catégorie de poids (g) } & \multirow{2}{*}{$\begin{array}{c}\text { 20-25 } \\
100\end{array}$} & \multirow{2}{*}{$\begin{array}{c}\mathbf{2 5 - 3 0} \\
37,5\end{array}$} & \multirow{2}{*}{$\begin{array}{r}\text { 30-35 } \\
12,7\end{array}$} & \multirow{2}{*}{$\begin{array}{c}\mathbf{3 5 - 4 0} \\
11,4\end{array}$} & \multirow{2}{*}{$\begin{array}{c}\text { 40-45 } \\
8,9\end{array}$} & \multirow{2}{*}{$\begin{array}{c}\mathbf{4 5 - 5 0} \\
7,5\end{array}$} & \multirow{2}{*}{$\begin{array}{c}\text { Ensemble } \\
29,7\end{array}$} \\
\hline OEufs & Clairs (\%) & & & & & & & \\
\hline & Fertiles (\%) & 0,0 & 62,5 & 87,3 & 88,6 & 91,1 & 92,5 & 70,3 \\
\hline Nb. de pintadeaux & & - & 56 & 237 & 965 & 531 & 113 & 1902 \\
\hline \multirow[t]{2}{*}{ Taux (\%) d'éclosion } & Apparent & - & 33,8 & 71,5 & 70,3 & 76,3 & 76,7 & 65,7 \\
\hline & Réel & - & 54,1 & 85,7 & 87,5 & 90,7 & 95,4 & 82,7 \\
\hline Ages (jours) & & \multicolumn{7}{|c|}{ Poids des pintadeaux (g) } \\
\hline \multirow[t]{2}{*}{1} & & - & $19,6^{\mathrm{a}}$ & $22,7^{b}$ & $25,2^{c}$ & $28,1^{d}$ & $30,6^{\mathrm{e}}$ & 25,6 \\
\hline & & - & $\pm 1,6$ & $\pm 1,4$ & $\pm 1,7$ & $\pm 1,4$ & $\pm 1,3$ & $\pm 2,6$ \\
\hline \multirow[t]{2}{*}{14} & & - & $21,8^{a}$ & $31,8^{b}$ & $42,8^{\mathrm{c}}$ & $52,1^{d}$ & $59,9^{\mathrm{e}}$ & 43,3 \\
\hline & & - & $\pm 1,8$ & $\pm 3,7$ & $\pm 7,7$ & $\pm 7,2$ & $\pm 8,9$ & $\pm 10,2$ \\
\hline \multirow[t]{2}{*}{28} & & - & $28,4^{\mathrm{a}}$ & $47,1^{b}$ & $65,6^{\mathrm{c}}$ & $82,2^{d}$ & $111,6^{\mathrm{e}}$ & 66,7 \\
\hline & & - & $\pm 5,7$ & $\pm 9,1$ & $\pm 8,8$ & $\pm 9,4$ & $\pm 12,6$ & $\pm 16,4$ \\
\hline \multirow[t]{2}{*}{42} & & - & $34,7^{\text {a }}$ & $66,0^{b}$ & $99,3^{\mathrm{c}}$ & $128,3^{d}$ & $181,4^{\mathrm{e}}$ & 101,3 \\
\hline & & - & $\pm 7,8$ & $\pm 12,6$ & $\pm 13,8$ & $\pm 14,1$ & $\pm 15,3$ & $\pm 27,8$ \\
\hline \multirow[t]{2}{*}{56} & & - & $47,5^{\mathrm{a}}$ & $100,7^{b}$ & $152,1^{\mathrm{c}}$ & $190,2^{d}$ & $271,8^{\mathrm{e}}$ & 153,2 \\
\hline & & - & $\pm 15,2$ & $\pm 17,3$ & $\pm 16,3$ & $\pm 22,6$ & $\pm 30,2$ & $\pm 39,9$ \\
\hline Taux de mortalité (\%) & & & 46,4 & 17,9 & 7,6 & 5,1 & 5,7 & 16,5 \\
\hline
\end{tabular}

Les différences entre les valeurs suivies de la même lettre en exposant sur la même ligne ne sont pas significatives au seuil de $5 \%$.

Le taux d'éclosion faible $(54,1 \%)$ des œufs de la catégorie de poids inférieur à $30 \mathrm{~g}$ a été comparable à celui rapporté par Okaeme (1986) et Ayorinde et al. (1988). Afin d'obtenir un taux d'éclosion intéressant, les œufs de moins de $30 \mathrm{~g}$ seraient donc à soustraire des opérations éventuelles d'incubation. Le taux réel d'éclosion de 82,7\% a été comparable à celui de 80,9\% rapporté par Veitsman et al. (1975), et de 81,0\% noté par Ayorinde et Ayeni (1986). Il a été supérieur au taux de $44 \%$ enregistré au Mali central par Kuit et al. (1986), et au taux de $77,45 \%$ noté au Nigeria par Ayorinde et al. (1988).

\section{Poids du pintadeau, croissance et mortalités}

Le poids vif moyen du pintadeau à l'éclosion a été de 25,6 $\pm 2,6 \mathrm{~g}$ pour l'ensemble des catégories. Ce poids vif a crû significativement ( $\mathrm{p}<0,05)$ avec le calibre de l'œuf. Il a été de 19,6 $\pm 1,6 \mathrm{~g}$ dans la catégorie des 25-30 g, et de 30,6 $\pm 1,3 \mathrm{~g}$ dans celle des 45-50 g (tableau II). Le poids vif moyen du pintadeau a représenté en moyenne 67,0\% du poids de l'œuf. Ce pourcentage a légèrement diminué au fur et à mesure que le poids de l'œuf augmentait. Il a été de 70,3\% dans la catégorie des $25-30 \mathrm{~g}$, de 70,1\% dans celle des 30-35 g et de $66,3 \%$ dans celle des 35-40 g. La valeur de 65,3\% a été enregistrée dans la catégorie des 40-45 g et la valeur de 64,7\% dans celle des $45-50 \mathrm{~g}$. Le poids vif du pintadeau à l'éclosion a été significativement corrélé au poids de l'œuf $(r=0,96, p<0,05)$. L'équation de régression linéaire entre le poids vif du pintadeau $(\mathrm{Y})$ à l'éclosion et le poids de l'œuf $(\mathrm{X})$ a été de la forme : $\mathrm{Y}=5,39+0,45 \mathrm{X}$ avec $\mathrm{R}^{2}=0,72$.

Le taux moyen de mortalité des pintadeaux de l'éclosion à huit semaines d'âge a diminué avec l'augmentation du poids de l'œuf (tableau II). Les taux de mortalité les plus bas ont été enregistrés dans les catégories de poids les plus élevés (respectivement $5,1 \%$ et $5,7 \%$ pour la catégorie des $40-45 \mathrm{~g}$ et des $45-50 \mathrm{~g})$. Il a été en revanche très élevé $(46,4 \%)$ pour la catégorie des œufs les plus légers. Il a été en moyenne de 16,5\% pour l'ensemble des catégories. Cette valeur s'explique principalement par le taux de mortalité important des pintadeaux de la catégorie des œufs de faible poids (25-30 g). Ainsi la valeur moyenne de mortalité a été ramenée à 9,1\% en excluant les données de cette catégorie.

La catégorie de poids de l'œuf a eu un effet significatif $(p<0,05)$ sur le poids vif du pintadeau aux âges types. Le poids vif moyen du pintadeau est passé de 43,3 $\pm 10,2 \mathrm{~g}$ à deux semaines d'âge, à $66,7 \pm 6,4 \mathrm{~g}$ à quatre semaines, à 101,3 $\pm 27,8 \mathrm{~g}$ à six semaines, puis à 153,2 $\pm 39,9 \mathrm{~g}$ à huit semaines (tableau II).

Les coefficients de corrélation (r) entre les poids vifs moyens des pintadeaux et la catégorie de poids des œufs ont présenté une moyenne de 0,94 . La catégorie de poids des œufs a eu un effet significatif $(\mathrm{p}<0,05)$ sur le poids vif des pintadeaux, quel qu'ait été l'âge des oiseaux. A deux semaines d'âge, il a été de 0,84 , à quatre semaines de 0,94 ,à six semaines de 0,95 et à huit semaines de 0,93 . L'équation de régression, qui lie le poids vif du pintadeau $(\mathrm{Y})$ à huit semaines d'âge à celui de l'œuf $(\mathrm{X})$, est donnée par la fonction: $\mathrm{Y}(\mathrm{g})=-279+11,8 \mathrm{X}(\mathrm{g})$ avec $\mathrm{R}^{2}=0,87$.

Les gains de poids moyens (gmq), calculés chaque deux semaines durant l'essai, sont représentés par le tableau III. La vitesse de croissance pondérale a augmenté significativement $(p<0,05)$ avec la catégorie de poids de l'œuf et l'âge du pintadeau. Le gmq de zéro à huit semaines a été de 4,3 $\pm 1,1 \mathrm{~g}$ pour l'ensemble des pintadeaux.

Abiola et al. (2008) rapportent que chez les poules les poussins les plus petits sont issus des œufs légers, tandis que les gros poussins proviennent des œufs plus lourds. Ces auteurs ont ainsi observé une corrélation positive entre le poids de l'œuf et celui du poussin. De même les résultats de Ramaphala et Mbajiorgu (2013) indiquent que les gros œufs produisent des poussins plus lourds $(\mathrm{p}<0,05)$ que ceux des poids moyens. Alkan et al. (2008) affirment que l'éclosabilité et le poids des cailles à l'éclosion, et par la suite les performances de croissance sont étroitement liés au poids de l'œuf. L'importance des réserves nutritives de l'œuf serait en faveur du meilleur développement de l'embryon. Cette relation est observée quel que soit l'âge de l'oiseau. Mbajiorgu (2011), cité par Moreki et Mothei (2013), note 
Tableau III

Gain de poids moyen des pintadeaux (Numida meleagris) selon la catégorie de poids de l'œuf

\begin{tabular}{lccccccc} 
Age (semaines) & $\mathbf{2 5 - 3 0}$ & $\mathbf{3 0 - 3 5}$ & $\mathbf{3 5 - 4 0}$ & $\mathbf{4 0 - 4 5}$ & $\mathbf{4 5 - 5 0}$ & Ensemble \\
\hline $0-2$ & 0,3 & 0,7 & 1,3 & 1,7 & 2,1 & 1,2 \\
$3-4$ & 0,5 & 1,1 & 1,6 & 2,2 & 4,0 & 1,8 \\
$5-6$ & 0,5 & 1,4 & 2,4 & 3,3 & 5,0 & 2,5 \\
$7-8$ & 0,9 & 2,5 & 3,8 & 4,4 & 6,5 & 3,6
\end{tabular}

l'effet de la taille des œufs d'incubation sur l'éclosabilité des œufs de poulets indigènes : l'éclosabilité a été plus fréquente pour les œufs de grande taille $(60-69 \mathrm{~g})$ que pour les œufs moyens $(<50-59 \mathrm{~g})$ et que pour les œufs de petite taille $(<49 \mathrm{~g})$ au seuil de signification de $5 \%$.

Il est bien établi que la mortalité des pintadeaux est l'un des problèmes majeurs de la méléagriculture au Burkina Faso. En effet, les taux de mortalité atteignent $89 \%$ (Bessin et al., 1998) et parfois $100 \%$ (Hien et al., 2000). Au Nigeria, Ayorinde et al. (1988), Oke et Ayorinde (1999), et Narushin et Romanov (2002) rapportent que la mortalité des pintadeaux est également importante $(60 \%)$. Nwagu et Alawa (1995) attribuent la forte mortalité des pintadeaux principalement au froid. La réduction du taux de mortalité embryonnaire à l'éclosion, lorsque le poids de l'œuf varie entre $30 \mathrm{~g}$ et $50 \mathrm{~g}$, pourrait s'expliquer par une meilleure vigueur des embryons, résultant d'un apport nutritionnel plus important.

\section{REFERENCES}

Abiola S.S., Meshioye O.O., Oyerinde B.O., Bamgbose M.A., 2008. Effect of egg size on hatchability of broiler chicks. Arch. Zootech., 57 (217): 83-86

Alkan S., Karabag K., Galic A., Balcioglu M.S., 2008. Effects of genotype and egg weight on hatchability traits and hatching weight in Japanese quail. South Afr. J. Anim. Sci., 38 (3)

Ayorinde K.L. Ayeni J.S.O., 1986. The reproductive performance of indigenous and exotic varieties of the guinea fowl (Numida meleagris) during different seasons in Nigeria. J. Anim. Prod. Res. 6 (2): 127-140

Ayorinde K.L., Oluyemi J.A., Ayeni J.S.O., 1988. Growth performance of four indigenous helmeted guinea fowl varieties (Numida meleagris galeata Pallas) in Nigeria. Bull. Anim. Health Prod. Afr., 36: 356-360

Bessin R., Belem A.M.G., Boussini H., Compaore Z., Kaboret Y., Dembélé M.A., 1998. Causes of young guinea fowl mortality in Burkina Faso. Rev. Elev. Med. Vet. Pays Trop., 51 (1): 87-93, doi: 10.19182/remvt.9658

Caglayan T.S., Alasahan K., Gunlu A., 2009. Effect of different egg storage periods on some egg quality characteristics and hatchability of partridges (Alectoris graeca). Poult. Sci., 88 (6): 1330-1333

Hien O.C., 2002. Effets de l'amélioration des conditions sanitaires sur le développement testiculaire, la LH et la ponte de la pintade locale au Burkina Faso. Thèse Doct., UFR-SVT, Université de Ouagadougou, Burkina Faso, $126 \mathrm{p}$

Hien O.C., Boly H., Diarra B., Sawadogo L., 2000. Influence du mode d'élevage sur la mortalité et la croissance des pintades en saison hivernale dans la zone sub-humide du Burkina Faso. Bull. Anim. Health Prod. Afr., $48: 236-245$

Kuit H.G., Traore A., Wilson R.T., 1986. Livestock production in Central Mali: ownership, management and productivity of poultry in traditional sector. Trop. Anim. Health Prod., 18: 222-231, doi: 10.1007/BF02359538

\section{CONCLUSION}

Le poids des œufs de pintade a été très variable, de la catégorie des 20-25 g à celle des $45-50 \mathrm{~g}$. Le maximum d'œufs a été noté dans la catégorie des 30-35 g. Les catégories de poids des œufs ont été corrélées positivement aux paramètres de reproduction : taux de fertilité et taux d'éclosion. Le poids de l'œuf a été positivement relié à celui du pintadeau et aux performances de croissances ultérieures de celui-ci. En outre, le taux de mortalité a été supérieur chez les animaux légers, provenant d'œufs légers. Le poids de l'œuf pourrait être un critère de sélection intéressant pour la pintade, aussi bien pour des raisons de performances de reproduction que pour les performances de croissance ultérieures des animaux. A court terme, on peut recommander d'écarter les œufs de poids inférieur à $35 \mathrm{~g}$ de la reproduction, et de les utiliser pour la consommation.
Mbajiorgu C.A., 2011. Effect of egg weight on hatchability and chick hatch weight of indigenous Venda chickens. Indian J. Anim. Sci., 45 (4): 300-304

Ministère de l'Economie et des Finances, 2011. Stratégie de croissance accélérée et de développement durable. Ouagadougou, Burkina Faso, 108 p.

Moreki J.C., Mothei K.M., 2013. Effect of egg size on hatchability of guinea fowl Keets. Int. J. Innov. Res. Sci. Eng. Technol., 2 (10)

Narushin V.G., Romanov M.N., 2002. Caractéristiques physiques et éclosabilité de l'œuf. World Poult. Sci. J., 58: 297-304

Nwagu B.I., Alawa C.B.I., 1995. Guinea fowl production in Nigeria. World Poult. Sci. J., 51: 261-270, doi: 10.1079/WPS19950018

Okaeme A.N., 1986. Diseases of economic importance in guinea fowl (N. meleagris). In: Proc. 11th Annu. Conf. Nigerian Society for Animal Production, Ahmadu Bello University, Zaria, 23-27 March, 64-68

Oke U.K., Ayorinde K.L., 1999. Effect of photo-induction on egg production characteristics in the Nigerian local guinea fowl (Numida meleagris galeata Pallas). In: Proc. 26th Annu. Conf. Nigerian Society for Animal Production, Enhancing livestock production in Nigeria, 337-339

Ramaphala N.O., Mbajiorgu C.A., 2013. Effect of egg weight on hatchability and chick hatch-weight of COBB 500 broiler chickens. Asian J. Anim. Vet. Adv., 8 (7): 885-892, doi: 10.3923/ajava.2013.885.892

Sahin E.H., Sengor E., Cetingul I.S., Yardimei M., 2009. Relationship between pre-incubation egg parameters from old breeder hens, egg hatchability and chick weight. J. Anim. Vet. Adv., 8: 115-119

Senapati P.K.K.G., Dask A.K., Chatterjee, 1996. Relationship between egg weight, shape index, fertility and hatchability of Japanese quail eggs. Environ. Ecol. Stat., 14: 547-577

Veitsman L., Derevyanchenko A., Mishin A., Mitskevich N., 1975. Incubation of guinea-fowl eggs [Russian]. Ptitsevodstvo (4): 31-32

Wilson H.R., 1991. Interrelationships of egg size, chick size and posthatching growth and hatchability. World Poult. Sci. J., 47: 5-20 


\section{Summary}

Sanfo R., Traoré F., Yougbare B., Ouali W. Effect of the egg weight of guinea fowl (Numida meleagris) on growth and reproduction parameters of chicks in Burkina Faso

Two thousand three hundred guinea fowl eggs collected at Gampela Station in Burkina Faso were weighed, then divided into six classes ranging from $20-25 \mathrm{~g}$ to $45-50 \mathrm{~g}$. The eggs were incubated and the following parameters were recorded: fertility and hatching rates, guinea fowl weight, mortality, and weight growth up to 56 days of age. The average egg weight was $35.2 \pm 1.3 \mathrm{~g}$ with unequal distribution in the six classes; the highest number of eggs was in class $35-40 \mathrm{~g}$. The average egg fertility rate was $84.4 \%$; it was correlated $(r=0.81$; $\mathrm{p}<0.05)$ to egg weight. The apparent hatching rate was $65.7 \%$ and the actual rate was $82.7 \%$; the latter was correlated $(r=0.85 ; p<0.05)$ to egg weight. The average hatching live weight of guinea fowl was $25.3 \pm 4.3 \mathrm{~g}$; it was also significantly related to egg weight $(r=0.96 ; p<0.05)$. The average mortality rate for guinea fowl was $16.5 \%$; it was related to the weight of the egg and therefore of the chick. The average weight of guinea fowl at 56 days (PDS56) was $152.5 \pm 20.3 \mathrm{~g}$; it was related to egg weight (EW) according to the regression: PDS56 $=-279.1+11.8 \mathrm{EW}$ with $\mathrm{R}^{2}=0.87$. This study shows that it is important to reject low-grade eggs $(<35 \mathrm{~g})$ in order to produce high-performance chicks.

Keywords: Numida meleagris, guinea fowl, egg characters, egg hatchability, fertility, growth, Burkina Faso

\section{Resumen}

Sanfo R., Traoré F., Yougbare B., Ouali W. Efecto del peso del huevo de la gallina de Guinea (Numida meleagris) sobre los parámetros de crecimiento y de reproducción de los pollos en Burkina Faso

Se recolectaron 2300 huevos de gallina de Guinea en la estación Gampela en Burkina Faso, se pesaron, luego dividieron en seis clases, con un rango de 20-25 g hasta 45-50 g. Los huevos fueron incubados y se recolectaron los siguientes parámetros: tasa de fertilidad y de eclosión, peso de los pollitos, mortalidad, y crecimiento en peso hasta los 56 días de edad. El peso promedio de los huevos fue de 35,2 $\pm 1,3 \mathrm{~g}$, con una distribución desigual entre las seis clases; el número más elevado de huevos estuvo en la clase 35-40 g. La tasa promedio de fertilidad de los huevos fue $84,4 \%$; estuvo correlacionada $(r=0,81 ; p<0,05)$ con el peso del huevo. La tasa de eclosión aparente fue de $65,7 \%$ y la tasa real fue de $82,7 \%$; esta última estuvo correlacionada $(r=0,85 ; p<0,05)$ con el peso del huevo. El peso vivo promedio a la eclosión de la gallina de Guinea fue de 25,3 \pm 4,3 g; estuvo también relacionado significativamente con el peso del huevo $(r=0,96 ; p<0,05)$. La tasa promedio de mortalidad de la gallina de Guinea fue de 16,5\%; estuvo relacionada con el peso del huevo y por lo tanto del pollo. El peso promedio de la gallina de Guinea a los 56 días (PDS56) fue de 152,5 $\pm 20,3$ g; estuvo relacionado con el peso del huevo $(\mathrm{PH})$ según la regresión: PDS56 $=-279,1+11,8 \mathrm{PH}$ con $\mathrm{R}^{2}=0,87$. Este estudio muestra que es importante rechazar los huevos de bajo peso $(<35 \mathrm{~g})$, con el fin de producir pollos de alto rendimiento.

Palabras clave: Numida meleagris, gallina de Guinea, características del huevo, eclosabilidad del huevo, fertilidad, crecimiento, Burkina Faso 
